\title{
Empathy in Medicine \\ Osteopathic and Allopathic Physician Interpersonal Manner, Empathy, and Communication Style and Clinical Status of Their Patients: A Pain Registry- Based Study
}

John C. Licciardone, DO, MS, MBA; Monika E. Schmitt, OMS III; Subhash Aryal, PhD

From the Department of

Family Medicine and The Osteopathic Research Center (Dr Licciardone), the Texas

College of Osteopathic

Medicine (Student Doctor Schmitt), and the Department

of Biostatistics and Epidemiology (Dr Aryal) at the University of North Texas Health Science Center in Fort Worth. Dr Licciardone is the

executive director of The Osteopathic Research Center and the Osteopathic Heritage Foundation Richards-Cohen Distinguished Chair in Clinical Research.

Financial Disclosures: None reported.

Support: The study was supported in part by grants to

Dr Licciardone from the Osteopathic Heritage Foundation and the American

Osteopathic Association.

Address correspondence to John C. Licciardone, DO, MS, MBA, 3500 Camp Bowie Blvd, Fort Worth, TX 76107-2644.

E-mail: john.licciardone@ unthsc.edu Submitted January 4, 2019; accepted February 13, 2019.
Context: Comparisons of osteopathic physicians (ie, DOs) and allopathic physicians (ie, MDs) on interpersonal manner, including empathy and communication style, have been limited by such methodologic issues as self-assessment and a focus on medical students rather than practicing physicians.

Objective: To compare perceptions of the interpersonal manner, empathy, and communication style of DOs and MDs and corresponding clinical measures reported by their patients.

Methods: A cross-sectional study of adults with subacute or chronic low back pain was conducted within the PRECISION Pain Research Registry from April 2016 through December 2018. A total of 313 patients having their physician for 1 year or longer reported sociodemographic and clinical characteristics, including use of nonsteroidal anti-inflammatory drugs (NSAIDs) and opioids for low back pain. Using validated research instruments, they also reported perceptions of their physician's interpersonal manner, empathy, and communication style and clinical measures of pain catastrophizing, pain self-efficacy, low back pain intensity, back-related disability, and deficits in quality of life relating to sleep disturbance, pain interference with activities, anxiety, depression, and low energy/fatigue.

Results: Patients treated by DOs were less likely to be using NSAIDs (odds ratio [OR], 0.60 ; 95\% CI, 0.36-0.997) or opioids (OR, 0.57; 95\% CI, 0.32-0.998) than patients treated by MDs. Patients treated by DOs reported lesser pain catastrophizing (mean, 12.5; 95\% CI, 10.1-15.0 for DOs vs 18.1 ; $95 \%$ CI, 16.3-19.9 for MDs; $P<.001)$ and greater pain self-efficacy (mean, 39.5; 95\% CI, 36.3-42.8 for DOs vs 35.3 ; 95\% CI, 33.4-37.3 for MDs; $P=.03$ ). Correspondingly, patients treated by DOs reported lesser back-related disability (mean, 11.2; 95\% CI, 9.9-12.5 for DOs vs 13.5 ; 95\% CI, 12.8 14.3 for MDs; $P=.002$ ) and a trend toward lesser deficits in quality of life. Patients reported more favorable perceptions of DOs on interpersonal manner (mean, 4.3; 95\% CI, 4.2-4.5 for DOs vs 4.0; 95\% CI, 3.9-4.2 for MDs; $P=.01$ ) and empathy (mean, 41.2; $95 \%$ CI, 39.1-43.3 for DOs vs 38.0; 95\% CI, 36.5-39.5 for MDs; $P=.02$ ).

Conclusion: The mechanisms underlying lesser use of NSAIDs and opioids, superior clinical status measures, and more favorable perceptions of physician interpersonal manner and empathy reported by patients treated by DOs warrant further investigation.

J Am Osteopath Assoc. 2019;119(8):499-510. Published online July 15, 2019 doi:10.7556/jaoa.2019.092

Keywords: low back pain, osteopathic manipulative treatment, pain research registry, physician empathy 
$\mathrm{T}$ he osteopathic philosophy is thought to be distinctive and maintained through a medical student selection and education process that values and fosters humanistic aspects of medical care as well as biomedical knowledge. This humanistic approach is reflected in the 4 principles of osteopathic philosophy ${ }^{1}$ : (1) the human being is a dynamic unit of function; (2) the body possesses selfregulatory mechanisms that are self-healing in nature; (3) structure and function are interrelated at all levels; and (4) rational treatment is based on these principles.

Osteopathic manipulative treatment has an integral role in optimizing the musculoskeletal system and, by extension, human biology and behavior. ${ }^{1}$ However, there is reason to believe that osteopathic physicians (ie, DOs) have a unique approach to managing musculoskeletal pain that transcends the use of osteopathic manipulative treatment or drug therapy. Findings from the National Ambulatory Medical Care Survey ${ }^{2}$ show that DOs provide a disproportionately large percentage of primary care for low back pain in the United States, while maintaining greater continuity of care and less often referring patients to other specialty physicians. Such interpersonal factors as physician empathy and communication style may be important determinants in seeking and retaining osteopathic medical care. Empathy and communication style also represent 2 aspects of the medical encounter that may contribute to patient satisfaction.

Thus far, few studies have compared DOs and allopathic physicians (ie, MDs) with regard to the processes of medical care that occur during physician encounters. Comparative studies of empathy have focused on osteopathic and allopathic medical students. $\mathrm{A} \mathrm{study}^{3}$ that compared self-reported student empathy levels found that allopathic medical students reported greater empathy than osteopathic medical students in the firstand second-year classes, but not in the third- and fourth-year classes. Alternatively, another study of selfreported empathy found a significant difference in favor of osteopathic medical students, ${ }^{4}$ but only among thirdyear students in comparison with an externally acquired control group. ${ }^{5}$ Such studies of medical student empathy are limited in several important ways: they measure only the student's self-assessment of empathy; the comparisons involve students from single osteopathic and allopathic institutions that are not generalizable to the broader populations of osteopathic and allopathic medical students; and they do not provide measures of empathy that reflect actual patient encounters with practicing physicians.

Validation is a physician communication style that focuses on understanding and empathy during patient encounters and involves such features as active listening, empathetic statements, and appropriate body language. These may be particularly important for patients with nonspecific low back pain who are often misunderstood, frustrated, and unsatisfied with their care. A randomized controlled trial found immediate improvements in ratings of sadness, anger, and frustration among patients with low back pain who were interviewed with a standardized validating communication style, but not in patients interviewed with an invalidating communication style. ${ }^{6}$

Key osteopathic principles were operationalized to develop a series of items to measure verbal communications that comprised typical patient-physician encounters in the Maine Osteopathic Outcomes Study. ${ }^{7}$ Therein, audiotaped patient encounters involving DOs and MDs were used to study visits considered to be more vs less consistent with "osteopathic intervention." Osteopathic physicians more often demonstrated a communication style that was reflective of osteopathic principles of practice, with the greatest DO-MD difference in communication observed in patients with low back pain. The investigators concluded that DOs were easily distinguishable from MDs by their verbal interactions with patients (particularly those having low back pain) but called for larger studies to replicate their findings.

In a recent systematic review, ${ }^{8}$ osteopathic treatment was shown to have a positive impact on such psychosocial factors as anxiety and fear avoidance. The review highlights the need for more research to elaborate on the mechanisms underlying the impact of osteopathic treatment. Measuring how such DO 
characteristics as interpersonal manner, empathy, and communication style may affect patient care and outcomes represents an important aspect of the needed research. Consequently, the purpose of the current study was to measure differences in the interpersonal manner, empathy, and communication style of DOs and MDs who deliver medical care for low back pain and to identify corresponding differences in clinical status measures reported by their patients.

\section{Methods}

\section{Patient Recruitment, Screening, and Enrollment}

Patients with subacute or chronic low back pain were recruited from the PRECISION Pain Research Registry (Pain Registry for Epidemiological, Clinical, and Interventional Studies and Innovation) at the University of North Texas Health Science Center during the period April 2016 through December 2018. A comprehensive overview of the registry, including procedures for collection of patient-reported data and biological specimens, has been published elsewhere. ${ }^{9}$ The Dallas-Fort Worth metroplex of over 7 million persons served as the catchment area for the registry during this period. Patients were screened for registry eligibility using a variety of methods. Registry staff screened patients within the group practice plan at the University of North Texas Health Science Center and at local community events, such as health fairs. Recruitment flyers were also distributed at local hospitals, clinics, and physician offices to direct patients to an online screening questionnaire. Conference presentations, print advertising, emails, and letters were used to seek physician referrals for patient screening. Direct-to-patient advertising through the registry's website and social media presence on Facebook and Twitter was also used to recruit patients. The research protocol was approved by the North Texas Regional Institutional Review Board. All eligible patients provided written informed consent prior to enrolling in the registry and participating in the study.

\section{Inclusion and Exclusion Criteria}

Patient inclusion criteria for this study were being aged 21 to 79 years; self-reporting low back pain for at least 2 months (subacute pain) or 6 months (chronic pain), with a frequency of pain on at least half of the days during the relevant period; being able to respond to data collection instruments in English; and having a physician who had provided their medical care for 1 year or longer. The inclusion criteria for chronic low back pain duration and frequency described herein match the case definition established by the National Institutes of Health (NIH) Task Force on Research Standards for Chronic Low Back Pain. ${ }^{10}$ Exclusion criteria were being pregnant (based on self-report) or being institutionalized. Patients received monetary compensation for their time and travel expenses to complete the baseline registry visit that included data for this study.

\section{Primary Measures Used to Characterize Patients}

The PRECISION Pain Research Registry routinely collects baseline data to characterize its patients and their clinical status. The relevant measures used in this study are described below.

\section{National Institutes of Health Minimum Dataset for Low Back Pain}

This instrument is recommended by the NIH Task Force on Research Standards for Chronic Low Back Pain to describe patients participating in research studies. ${ }^{10}$ It includes 40 patient-reported items, including sociodemographic characteristics, medical history, symptoms, functioning, and interventions for low back pain. These items were adapted to be relevant to patients with either subacute or chronic low back pain.

\section{Numerical Rating Scale for Low Back Pain Intensity}

This scale measured low back pain "on average over the past 7 days" using an 11-point scale ranging from 0 ("no pain") to 10 ("worst pain"). 


\section{Roland-Morris Disability Questionnaire}

The Roland-Morris Disability Questionnaire consists of 24 items that assess back-related disability by measuring how much low back pain adversely affects patient functioning and activities. ${ }^{11}$ Each item was scored as either 1 ("agree" that low back pain has an adverse impact) or 0 ("disagree" that low back pain has an adverse impact). This questionnaire is scored as the sum of responses to each item, thereby potentially ranging from 0 to 24 .

\section{Quality of Life Deficits}

The 29-item Patient-Reported Outcomes Measurement Instrument System (PROMIS-29) is a quality of life measure that includes items derived from the PROMIS pain behavior item bank that assesses how low back pain interferes with normal activities and assesses levels of anxiety, depression, fatigue, and sleep disturbance. $^{12}$ The SPADE cluster (sleep disturbance, pain interference with activities, anxiety, depression, and low energy/fatigue scales) was used as a composite measure in this study. Scores for each scale of the SPADE cluster are normed based on the United States general population such that the mean and SD are 50 and 10, respectively. The overall SPADE cluster score was then computed as the mean of its 5 component scales, with higher scores representing greater quality of life deficits.

\section{History of Medical Conditions Inventory}

This inventory consists of 10 back-related or general medical conditions that patients may report ever having, including herniated disc, sciatica, osteoarthritis, osteoporosis, hypertension, heart disease, diabetes mellitus, asthma, bronchitis, and depression.

\section{Pain Catastrophizing Scale}

This scale measures the phenomenon defined as an exaggerated negative mental set brought to bear during actual or anticipated painful experiences. ${ }^{13}$ It consists of 13 ordinal scale items comprising the 3 dimensions of rumination, magnification, and helplessness. ${ }^{14}$
The total score ranges from 0 (no catastrophizing) to 52 (greatest level of catastrophizing).

\section{Pain Self-Efficacy Questionnaire}

This questionnaire measures the degree to which patients mitigate pain and the negative emotions associated with it to maintain everyday life activities, including work. ${ }^{15}$ It consists of 10 ordinal scale items that assess confidence in performing various activities. ${ }^{16}$ The total score ranges from 0 (no self-efficacy) to 60 (greatest level of self-efficacy).

\section{Primary Measures of Physician Interpersonal Manner, Empathy, and Communication Style}

Validated research instruments were used as primary measures of patient perceptions of their physician's interpersonal manner, empathy, and communication style. These measures are described below.

\section{Interpersonal Manner}

Physician interpersonal manner was measured with the 18-item Patient Satisfaction Questionnaire, ${ }^{17}$ which is a shorter version of the questionnaire that was originally developed to measure satisfaction with medical care in a general population. ${ }^{18}$ The interpersonal manner scale comprises 2 items that are individually scored and then averaged to compute the scale score. Scale scores potentially range from 1 to 5 , with higher scores representing greater satisfaction with the interpersonal manner of the physician. The Cronbach $\alpha$ for the scale is 0.66 .

\section{Consultation and Relational Empathy Measure}

This instrument was developed and validated as a process measure of empathy during physician encounters for primary care. ${ }^{19}$ It is applicable to virtually all clinical encounters and is not influenced by the patient's chief complaint, socioeconomic status, chronicity of disease, or emotional state. ${ }^{20}$ The measurement consists of 10 items that are individually scored and then totaled to compute the overall score, which may 
potentially range from 10 to 50 . Higher scores represent more favorable patient perceptions of physician empathy. The Cronbach $\alpha$ for the measure is 0.93 .

\section{Communication Behavior Questionnaire}

This questionnaire was developed and validated as a measure of patient preferences with regard to physician communications relating to low back pain. ${ }^{21}$ It consists of 23 items that represent 4 scales, with the Cronbach $\alpha$ for the scales ranging from 0.88 to 0.92 . Items on each scale are scored and then transformed to compute the overall score across all 4 scales, which potentially ranges from 0 to 100 . Higher scores reflect more desirable physician communication behavior.

\section{Data Management and Statistical Analysis}

Patients completed the data collection instruments using a computer or mobile device provided by project personnel and the Qualtrics Survey Software. Research coordinators assisted patients if they had difficulty reading or understanding the questions. The electronic data collection system permitted patients to take breaks while answering questions if needed; however, it required completion of all items within the research instruments. Qualtrics survey data were exported to the IBM SPSS version 23 software, which was subsequently used for data management and analysis. Descriptive statistics were computed using numbers and percentages for nominal, dichotomous, and ordinal variables, and mean (SD) for continuous variables. Statistical comparisons of patients treated by DOs and MDs were performed using contingency table methods, including odds ratios (ORs) and 95\% CIs, or the $t$ test, as appropriate. Hypotheses were tested at the .05 level of statistical significance.

\section{Results}

\section{Sociodemographic Characteristics of Patients}

A total of 313 of the 488 registry patients (64.1\%) were eligible for the study (Figure 1). Patients ranged from

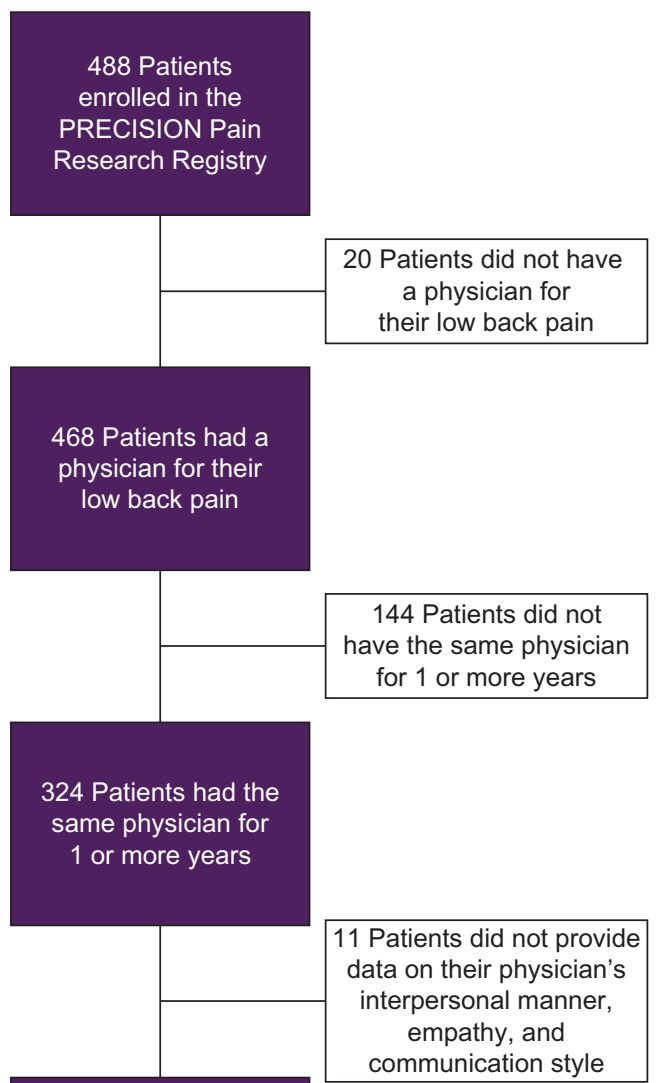

313 Patients provided

information on their

physician's interpersonal

manner, empathy, and

communication style

Figure 1.

Flowchart of patients eligible for inclusion in the pain registry-based study comparing interpersonal manner, empathy, and communication style between osteopathic and allopathic physicians. The numbers of patients excluded at each stage are presented. During initial stages of registry development, patients were not required to have a physician for their low back pain, and data were not collected on physician interpersonal manner, empathy, and communication style.

21 to 79 years of age, with a mean (SD) age of 54.7 (11.9) years. A total of 220 patients $(70.3 \%)$ were women. Osteopathic physicians treated 87 study patients $(31.9 \%)$. There were no statistically significant differences in the sociodemographic characteristics of patients treated by DOs or MDs (Table 1). 
Table 1.

Sociodemographic Characteristics of Patients With Low Back Pain Treated by Osteopathic or Allopathic Physicians $(\mathbf{N}=313)^{a}$

\begin{tabular}{|c|c|c|c|}
\hline Characteristic & $\begin{array}{c}\text { Osteopathic } \\
\text { Physicians (n=87) }\end{array}$ & $\begin{array}{c}\text { Allopathic } \\
\text { Physicians }(n=226)\end{array}$ & P Value \\
\hline Age, y (mean, [SD]) & $54.7(13.2)$ & $54.7(11.4)$ & .98 \\
\hline Sex & & & .11 \\
\hline Female & $67(77.0)$ & $153(67.7)$ & \\
\hline Male & $20(23.0)$ & $73(32.3)$ & \\
\hline Race & & & .14 \\
\hline White & $68(78.2)$ & $145(64.2)$ & \\
\hline Black & $18(20.7)$ & $72(31.9)$ & \\
\hline Asian & $1(1.1)$ & $3(1.3)$ & \\
\hline American Indian/Alaska Native & $0(0.0)$ & $4(1.8)$ & \\
\hline Native Hawaiian/Pacific Islander & $0(0.0)$ & $2(0.9)$ & \\
\hline Ethnicity & & & .27 \\
\hline Hispanic & $7(8.0)$ & $28(12.4)$ & \\
\hline Non-Hispanic & $80(92.0)$ & $198(87.6)$ & \\
\hline Educational Level & & & .13 \\
\hline High school diploma or below & $11(12.6)$ & $56(24.8)$ & \\
\hline Some college or post-high school education & $38(43.7)$ & $87(38.5)$ & \\
\hline Bachelor or Master degree & $35(40.2)$ & $75(33.2)$ & \\
\hline Doctoral or professional degree & $3(3.4)$ & $8(3.5)$ & \\
\hline Employment Status & & & .20 \\
\hline Working & $35(40.2)$ & $93(41.2)$ & \\
\hline Unemployed & $4(4.6)$ & $17(7.5)$ & \\
\hline Disabled because of low back pain & $7(8.0)$ & $26(11.5)$ & \\
\hline Disabled for other reasons & $10(11.5)$ & $37(16.4)$ & \\
\hline Retired or not looking for work & $31(35.6)$ & $53(23.5)$ & \\
\hline $\begin{array}{l}\text { Ever Applied for or Received Disability or Workers' } \\
\text { Compensation Benefits Because Was Unable to } \\
\text { Work Due to Low Back Pain }\end{array}$ & & & .59 \\
\hline Yes & $17(19.5)$ & $55(24.3)$ & \\
\hline No & $61(70.1)$ & $145(64.2)$ & \\
\hline Not applicable & $9(10.3)$ & $26(11.5)$ & \\
\hline Ever Involved in a Legal Claim Related to Low Back Pain & & & .73 \\
\hline Yes & $7(8.0)$ & $21(9.3)$ & \\
\hline No & $80(92.0)$ & $205(90.7)$ & \\
\hline
\end{tabular}

a Data are presented as No.(\%) unless otherwise indicated. 


\section{Clinical Characteristics of Patients}

A total of 280 patients $(89.5 \%)$ reported having chronic low back pain, and 72 (23.0\%) were substantially bothered by widespread pain over the past 4 weeks (Table 2). Depression had been diagnosed in 147 patients $(47.0 \%)$, hypertension in 131 patients (41.9\%), herniated disc in 104 patients $(33.2 \%)$, and sciatica in 103 patients $(32.9 \%)$. Nonsteroidal antiinflammatory drugs and opioids were used for low back pain by $210(67.1 \%)$ and $103(32.9 \%)$ patients, respectively. Exercise therapy and massage therapy were used by $180(57.5 \%)$ and 155 (49.5\%) patients, respectively. Patients treated by DOs had more favorable cigarette smoking histories, were less likely to be bothered by widespread pain, and were more likely to have been treated with spinal manipulation for low back pain. Patients treated by DOs were also less likely to be using NSAIDs (OR, 0.60; 95\% CI, 0.36-0.997) or opioids (OR, 0.57; 95\% CI, 0.32-0.998) for their low back pain.

\section{Clinical Status of Patients Relating to Low Back Pain}

The mean (SD) overall patient scores for pain catastrophizing and pain self-efficacy were 16.6 (13.5) and 36.5 (15.0), respectively. Patients reported mean (SD) scores of 5.8 (2.0) for low back pain intensity; 12.9 (6.0) for back-related disability; and 55.4 (6.8) for quality of life deficits. These clinical status measures reflect a study population that was moderately to severely impacted by low back pain. Patients treated by DOs reported significantly lesser pain catastrophizing and greater pain self-efficacy than patients treated by MDs (Figure 2). Patients treated by DOs also reported significantly lesser back-related disability and a trend toward lesser quality of life deficits.

\section{Interpersonal Manner, Empathy, and Communication Style of Physicians}

The mean (SD) overall physician scores were 4.1 (0.9) for interpersonal manner; 38.9 (11.1) for empathy; and 66.8 (22.0) for communication style. Osteopathic physicians were perceived significantly more favorably by their patients on the measures of interpersonal manner and empathy (Figure 3).

\section{Discussion}

This study found significant differences between DOs and MDs in measures of interpersonal manner and empathy reported by their patients. There were also significant differences favoring DOs in the measures of pain catastrophizing, pain self-efficacy, and back-related disability reported by their patients. Importantly, patients treated by DOs also reported greater use of spinal manipulation and lesser use of NSAIDs and opioids for low back pain than patients treated by MDs. Although a causal relationship between these physician characteristics and their patient's reported low back pain treatments and clinical status measures cannot be established because of the cross-sectional design used in this study, the findings suggest several questions about osteopathic medical care that warrant further investigation.

An important question involves the association of patient pain catastrophizing and pain self-efficacy with low back pain outcomes. Are pain catastrophizing and pain self-efficacy intrinsic patient characteristics that are essentially fixed, or are they susceptible to intervention? If pain catastrophizing can be attenuated and pain selfefficacy augmented, what is the role and impact of the osteopathic physician in these processes, and are such factors as physician interpersonal manner, empathy, and communication style important? Furthermore, independent of the issue of stability of pain catastrophizing and pain self-efficacy, does either factor mediate the relationship between the medical care provided by an osteopathic physician and the patient's clinical status over time? Finally, are there other important factors that complicate the relationship between the processes and outcomes of osteopathic medical care for low back pain? Unknown or uncontrolled confounders may distort the relationship between osteopathic physician characteristics, such as interpersonal manner and empathy, and such outcome variables as pain, functioning, and quality of life. For example, a study ${ }^{22}$ of beneficiaries in the Medicare 
Table 2.

Clinical Characteristics of Patients With Low Back Pain Treated by Osteopathic or Allopathic Physicians $(\mathbf{N}=\mathbf{3 1 3})^{\mathrm{a}}$

\begin{tabular}{|c|c|c|c|}
\hline Characteristic & $\begin{array}{c}\text { Osteopathic } \\
\text { Physicians (n=87) }\end{array}$ & $\begin{array}{c}\text { Allopathic } \\
\text { Physicians }(n=226)\end{array}$ & P Value \\
\hline Cigarette Smoking Status & & & .02 \\
\hline Never smoked & $54(62.1)$ & $104(46.0)$ & \\
\hline Former smoker & $25(28.7)$ & $80(35.4)$ & \\
\hline Current smoker & $8(9.2)$ & $42(18.6)$ & \\
\hline \multicolumn{4}{|l|}{ Medical Conditions Ever Diagnosed } \\
\hline Herniated disc & $28(32.2)$ & $76(33.6)$ & .81 \\
\hline Sciatica & $30(34.5)$ & $73(32.3)$ & .71 \\
\hline Osteoarthritis & $22(25.3)$ & $77(34.1)$ & .13 \\
\hline Osteoporosis & $11(12.6)$ & $19(8.4)$ & .25 \\
\hline Hypertension & $32(36.8)$ & $99(43.8)$ & .26 \\
\hline Heart disease & $10(11.5)$ & $23(10.2)$ & .73 \\
\hline Diabetes mellitus & $16(18.4)$ & $56(24.8)$ & .23 \\
\hline Asthma & $20(23.0)$ & $59(26.1)$ & .57 \\
\hline Bronchitis & $9(10.3)$ & $24(10.6)$ & .94 \\
\hline Depression & $41(47.1)$ & $106(46.9)$ & .97 \\
\hline Chronicity of Low Back Pain & & & .73 \\
\hline Subacute & $10(11.5)$ & $23(10.2)$ & \\
\hline Chronic & 77 (88.5) & $203(89.8)$ & \\
\hline Bothersomeness of Widespread Pain in the Past 4 Weeks & & & .02 \\
\hline Not at all & $43(49.4)$ & $74(32.7)$ & \\
\hline A little bit & $26(29.9)$ & $98(43.4)$ & \\
\hline A lot & $18(20.7)$ & $54(23.9)$ & \\
\hline History of Low Back Surgery & & & .76 \\
\hline Yes, one surgery & $8(9.2)$ & $16(7.1)$ & \\
\hline Yes, more than one surgery & $5(5.7)$ & $16(7.1)$ & \\
\hline No & $74(85.1)$ & $194(85.8)$ & \\
\hline Length of Relationship With Current Physician & & & .06 \\
\hline At least 1 year, but less than 5 years & $61(70.1)$ & $132(58.4)$ & \\
\hline 5 or more years & $26(29.9)$ & $94(41.6)$ & \\
\hline \multicolumn{4}{|l|}{ Nonpharmacologic Treatments Ever Used for Low Back Pain } \\
\hline Exercise therapy & $46(52.9)$ & $134(59.3)$ & .30 \\
\hline Massage therapy & $47(54.0)$ & $108(47.8)$ & .32 \\
\hline Yoga & $31(35.6)$ & $57(25.2)$ & .07 \\
\hline
\end{tabular}


Table 2 (continued).

Clinical Characteristics of Patients With Low Back Pain Treated by Osteopathic or Allopathic Physicians $(\mathbf{N}=\mathbf{3 1 3})^{\mathrm{a}}$

\begin{tabular}{lccc} 
Characteristic & $\begin{array}{c}\text { Osteopathic } \\
\text { Physicians (n=87) }\end{array}$ & $\begin{array}{c}\text { Allopathic } \\
\text { Physicians (n=226) }\end{array}$ & P Value \\
\hline Treatment & & & \\
\hline Spinal manipulation & $53(60.9)$ & $84(37.2)$ & $<.001$ \\
\hline Acupuncture & $19(21.8)$ & $33(14.6)$ & .12 \\
\hline Cognitive behavioral therapy & $7(8.0)$ & $19(8.4)$ & .92 \\
\hline Currently Using NSAIDs For Low Back Pain & & & .048 \\
\hline Yes & $51(58.6)$ & $159(70.4)$ & .048 \\
\hline No & $36(41.4)$ & $67(29.6)$ & \\
\hline Currently Using Opioids For Low Back Pain & & & \\
\hline Yes & $21(24.1)$ & $145(64.2)$ & \\
\hline No & $66(75.9)$ & & \\
\hline
\end{tabular}

a Data are presented as No. (\%) unless otherwise indicated.

Abbreviation: NSAID, nonsteroidal anti-inflammatory drug

inpatient and carrier files found that physician sex may affect such major outcomes as hospital mortality and readmission rates. More research, including longitudinal follow-up, is needed to further explore and answer questions relating to intrinsic osteopathic characteristics that may affect clinical outcomes for low back pain.

Recent clinical practice guidelines for low back pain from the Centers for Disease Control and Prevention ${ }^{23}$ and the American College of Physicians ${ }^{24}$ emphasize the importance of initiating management with nonpharmacologic treatments and avoiding opioid therapy unless the benefits outweigh the risks for a given patient. However, successful implementation of such recommendations may depend on the relationship between the physician and patient during medical encounters for low back pain. The findings of our study suggest that an "osteopathic practice style" (corroborated by such empirical findings as more favorable patient perceptions of interpersonal manner and empathy) may facilitate implementing the recommended treatment guidelines for low back pain. Not surprisingly, patients treated by DOs reported using spinal manipulation more frequently than patients treated by MDs. We presume that most spinal manipulation used for patients treated by DOs was osteopathic manipulative treatment, whereas spinal manipulation used for patients treated by MDs may more likely have been provided by chiropractors or physical therapists. Our findings demonstrate that patients treated by DOs more often reported low back pain management that was in accord with current clinical practice guidelines, including greater use of nonpharmacologic treatments (eg, spinal manipulation) and lesser use of NSAIDs and opioids, than patients treated by MDs.

The Centers for Disease Control and Prevention recently highlighted the prevalence of high-impact chronic pain in the United States, noting that about 20 million adults have such pain that interferes with work or life most days or every day. ${ }^{25}$ It is noteworthy that osteopathic medical care had a greater effect on backrelated disability than on low back pain intensity. This finding suggests that an osteopathic practice style and approach to pain management may help meet the Healthy People $2020^{26}$ developmental objective to decrease the prevalence of adults with high-impact chronic pain. 

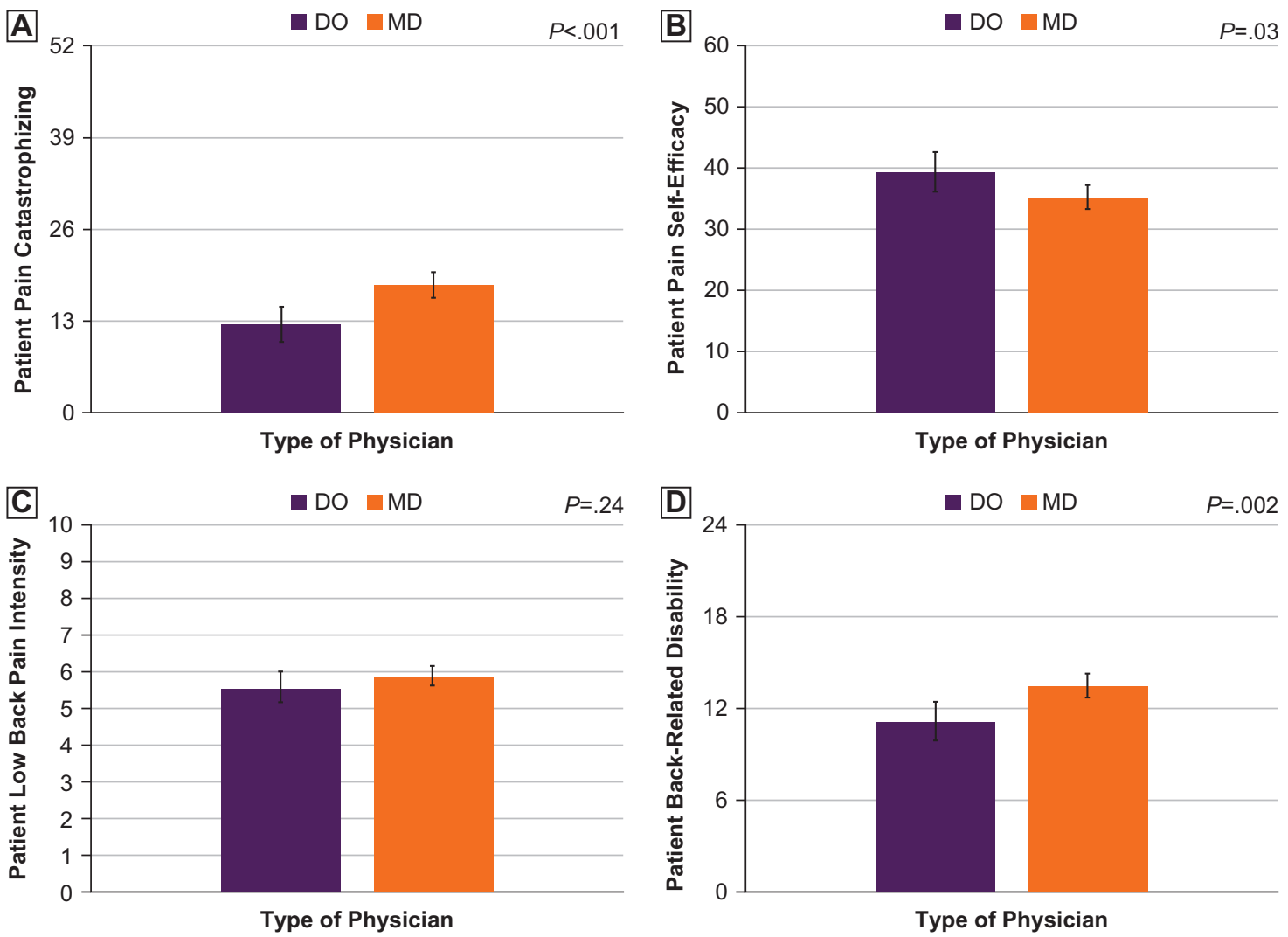

Type of Physician $P=.08$

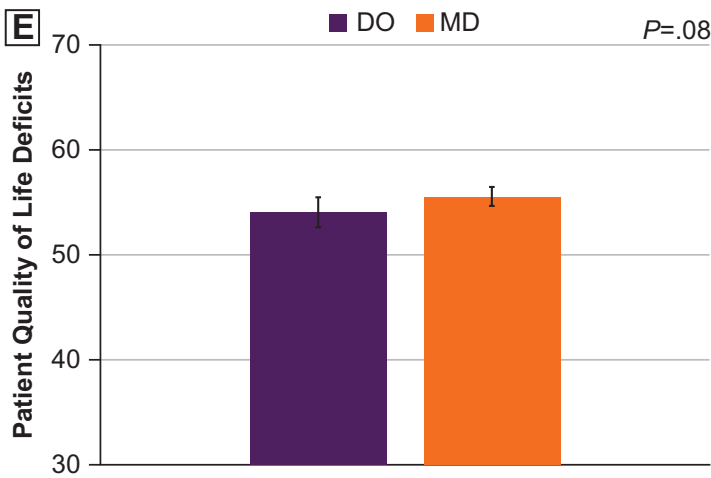

Type of Physician

Figure 2.

Patient-reported means of pain catastrophizing (A), pain self-efficacy (B), low back pain intensity (C), back-related disability (D), and quality of life deficits (E) by type of physician. The error bars represent the $95 \%$ Cls. Pain catastrophizing was measured with the Pain Catastrophizing Scale. Pain self-efficacy was measured with the Pain Self-Efficacy Questionnaire. Low back pain intensity was measured with a numerical rating scale. Back-related disability was measured with the Roland-Morris Disability Questionnaire. Quality of life deficits were measured with the SPADE cluster (sleep disturbance, pain interference with activities, anxiety, depression, low energy/fatigue) derived from the 29-item Patient-Reported Outcomes Measurement Information System. Higher scores represent less favorable clinical status on each measure except pain self-efficacy. Abbreviations: DO, osteopathic physician; MD, allopathic physician. 
There were several strengths of our study. Patients were recruited from a community-based pain research registry, thereby enhancing the representativeness of the study population. Recommendations of the NIH Task Force on Research Standards for Chronic Low Back Pain were adopted in conducting our study, including use of the case definition for chronic low back pain and clinical measures pertaining to low back pain intensity, back-related disability, and quality of life. Validated research instruments were used to measure patient perceptions of physician interpersonal manner, empathy, and communication style, as well as pain catastrophizing and pain self-efficacy. The study was adequately powered to detect significant differences in physician interpersonal manner, empathy, and communication style. For example, DO-MD differences of $0.5 \mathrm{SD}$ or greater on any of these 3 measures were detectable with greater than $95 \%$ statistical power.

The greatest limitations of the study were its observational cross-sectional design and lack of randomization. The cross-sectional design did not enable us to definitively assess causation because of the unclear temporal relationship between exposure to osteopathic medical care and patient clinical status measures. We mitigated this limitation by including only patients who reported having their physician for at least 1 year, thereby allowing time for clinical status to respond to the medical care provided. Lack of randomization did not appear to be an important source of bias, as patients treated by DOs or MDs were not significantly different from one another on important baseline variables, including sociodemographic characteristics, diagnosed medical conditions, chronicity of low back pain, or previous low back surgery.

\section{Conclusion}

The more favorable perceptions of DOs relating to interpersonal manner and empathy reported in this study, coupled with the lesser use of NSAIDs and opioids and superior clinical status measures reported by their patients, warrant further investigation to assess the mechanisms whereby osteopathic medical care may affect patient health. Productive areas of investigation
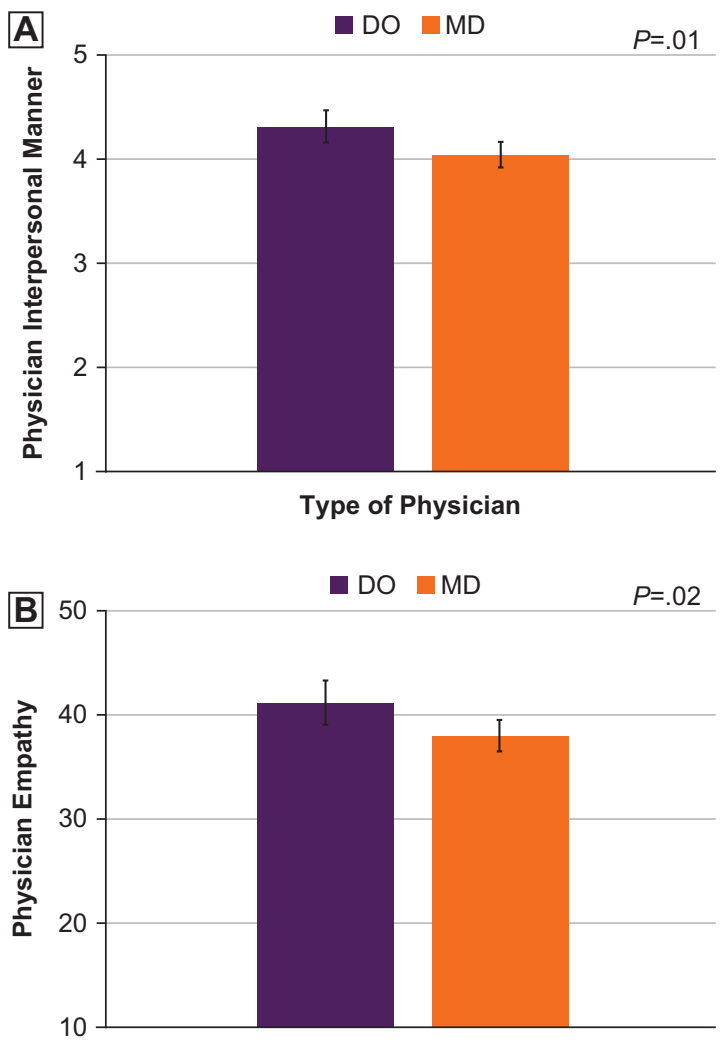

Type of Physician

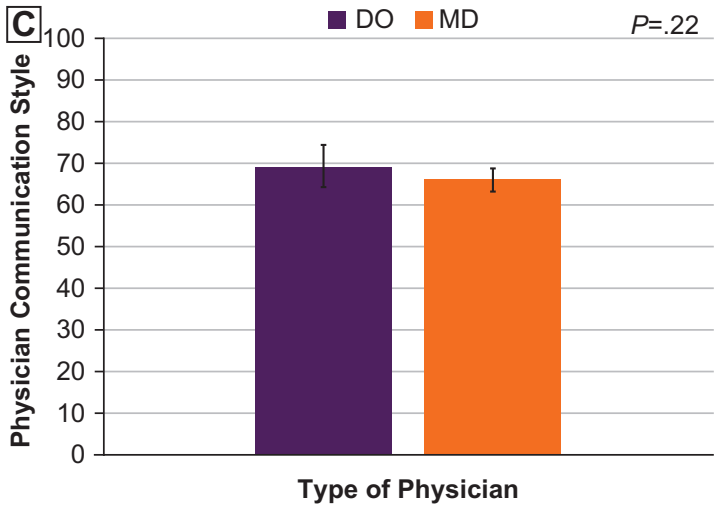

Figure 3.

Patient-reported mean perceptions of physician interpersonal manner $(A)$, physician empathy $(B)$, and physician communication style (C) by type of physician. The error bars represent the 95\% Cls. Interpersonal manner was measured with the scale on the 18-item Patient Satisfaction Questionnaire. Empathy was measured with the Consultation and Relational Empathy measure, and communication style was measured with the Communication Behavior Questionnaire. Higher scores represent more favorable perceptions on each measure. Abbreviations: $\mathrm{DO}$, osteopathic physician; MD, allopathic physician. 
may involve longitudinal follow-up of patients with low back pain treated by DOs or MDs for changes in clinical status over time and other studies to disentangle the various effects of osteopathic medical care to better understand the factors that represent mediators, moderators, and confounders in that process.

\section{Acknowledgments}

We thank the staff of the PRECISION Pain Research Registry and the patients enrolled in the registry for their contributions to this study.

\section{Author Contributions}

All authors provided substantial contributions to conception and design, acquisition of data, or analysis and interpretation of data; all authors drafted the article or revised it critically for important intellectual content; all authors gave final approval of the version of the article to be published; and all authors agree to be accountable for all aspects of the work in ensuring that questions related to the accuracy or integrity of any part of the work are appropriately investigated and resolved.

\section{References}

1. Seffinger MA, Hruby R, Rogers FJ, et al. Philosophy of Osteopathic Medicine. In: Seffinger MA, executive ed. Foundations of Osteopathic Medicine. 4th ed. Baltimore, MD: Wolters Kluwer; 2019:2-17.

2. Licciardone JC. A national study of primary care provided by osteopathic physicians. J Am Osteopath Assoc. 2015;115 (12):704-713. doi:10.7556/jaoa.2015.145

3. Kimmelman M, Giacobbe J, Faden J, Kumar G, Pinckney CC, Steer R. Empathy in osteopathic medical students: a cross-sectional analysis. J Am Osteopath Assoc. 2012;112(6):347-355.

4. Calabrese LH, Bianco JA, Mann D, Massello D, Hojat M. Correlates and changes in empathy and attitudes toward interprofessional collaboration in osteopathic medical students. J Am Osteopath Assoc. 2013;113(12):898-907. doi:10.7556/jaoa.2013.068

5. Chen D, Lew R, Hershman W, Orlander J. A cross-sectional measurement of medical student empathy. J Gen Intern Med. 2007;22 (10):1434-1438. doi:10.1007/s11606-007-0298-x

6. Vangronsveld $\mathrm{KL}$, Linton SJ. The effect of validating and invalidating communication on satisfaction, pain and affect in nurses suffering from low back pain during a semi-structured interview. Eur J Pain. 2012;16 (2):239-246. doi:10.1016/j.ejpain.2011.07.009

7. Carey TS, Motyka TM, Garrett JM, Keller RB. Do osteopathic physicians differ in patient interaction from allopathic physicians? an empirically derived approach. J Am Osteopath Assoc. 2003;103(7):313-318.

8. Saracutu M, Rance J, Davies H, Edwards DJ. The effects of osteopathic treatment on psychosocial factors in people with persistent pain: a systematic review. Int J Osteopath Med. 2018;27:23-33.

9. Licciardone JC, Gatchel RJ, Phillips N, Aryal S. The Pain Registry fo Epidemiological, Clinical, and Interventional Studies and Innovation (PRECISION): registry overview and protocol for a propensity score-matched study of opioid prescribing in patients with low back pain. J Pain Res. 2018;11:1751-1760.
10. Deyo RA, Dworkin SF, Amtmann D, et al. Report of the NIH Task Force on research standards for chronic low back pain. J Pain. 2014;15 (6):569-585. doi:10.1016/j.jpain.2014.03.005

11. Roland M, Morris R. A study of the natural history of back pain. Part I: development of a reliable and sensitive measure of disability in low-back pain. Spine. 1983;8(2):141-144.

12. Revicki DA, Chen WH, Harnam N, et al. Development and psychometric analysis of the PROMIS pain behavior item bank. Pain. 2009;146(1-2):158-169. doi:10.1016/j.pain.2009.07.029

13. Sullivan MJ, Thorn B, Haythornthwaite JA, et al. Theoretical perspectives on the relation between catastrophizing and pain. Clin J Pain. 2001;17(1):52-64

14. Sullivan MJ. The Pain Catastrophizing Scale: User Manual. Montreal, QC: McGill University; 2009.

15. Miles CL, Pincus T, Carnes D, Taylor SJ, Underwood M. Measuring pain self-efficacy. Clin J Pain. 2011;27(5):461-470.

16. Nicholas MK. The pain self-efficacy questionnaire: taking pain into account. Eur J Pain. 2007;11(2):153-163.

17. Marshall GN, Hays RD. The Patient Satisfaction Questionnaire Short-Form (PSQ-18). Santa Monica, CA: RAND Corp; 1994.

18. Ware JE, Snyder MK, Wright WR, Davies AR. Defining and measuring patient satisfaction with medical care. Eval Program Plann. 1983:6:247-263.

19. Mercer SW, Maxwell M, Heaney D, Watt GC. The consultation and relational empathy (CARE) measure: development and preliminary validation and reliability of an empathy-based consultation process measure. Fam Pract. 2004;21(6):699-705.

20. Mercer SW, McConnachie A, Maxwell M, Heaney D, Watt GC. Relevance and practical use of the consultation and relational empathy (CARE) measure in general practice. Fam Pract. 2005;22(3):328-334.

21. Farin E, Gramm L, Schmidt E. Taking into account patients' communication preferences: instrument development and results in chronic back pain patients. Patient Educ Couns. 2012;86 (1):41-48.

22. Tsugawa $Y$, Jena AB, Figueroa JF, Orav EJ, Blumenthal DM, Jha AK Comparison of hospital mortality and readmission rates for medicare patients treated by male vs female physicians. JAMA Intern Med. 2017;177(2):206-213. doi:10.1001/jamainternmed.2016.7875

23. Dowell D, Haegerich TM, Chou R. CDC guideline for prescribing opioids for chronic pain — United States, 2016. MMWR Recomm Rep. 2016;65(1):1-49. doi:10.15585/mmwr.rr6501e1

24. Qaseem A, Wilt TJ, McLean RM, Forciea MA, Clinical Guidelines Committee of the American College of Physicians. Noninvasive treatments for acute, subacute, and chronic low back pain: a clinical practice guideline from the American College of Physicians. Ann Intern Med. 2017;166(7):514-530.

25. Dahlhamer J, Lucas J, Zelaya C, et al. Prevalence of chronic pain and high-impact chronic pain among adults - United States, 2016. MMWR Morb Mortal Wkly Rep. 2018;67(36):1001-1006.

26. Arthritis, osteoporosis, and chronic back conditions: developmental objective AOCBC-13. US Department of Health and Human Services website. https://www.healthypeople.gov/2020/topics-objectives/topic/ arthritis-osteoporosis-and-chronic-back-conditions/objectives. Accessed September 24, 2018.

(C) 2019 American Osteopathic Association 